\title{
Comparative Analysis of Highly Transmitting Phase Correcting Structures for Electromagnetic Bandgap Resonator Antenna
}

\author{
Touseef Hayat \\ School of Engineering \\ Macquarie University \\ Sydney, Australia \\ touseef.hayat@students.mq.edu.au
}

\author{
Muhammad U.Afzal \\ School of Engineering \\ Macquarie University \\ Sydney, Australia \\ muhammad.afzal@mq.edu.au
}

\author{
Ali Lalbakhsh \\ School of Engineering \\ Macquarie University \\ Sydney, Australia \\ ali.lalbakhsh@students.mq.edu.au
}

\author{
Foez Ahmed \\ School of Engineering \\ Macquarie University \\ Sydney, Australia \\ foez.ahmed@students.mq.edu.au
}

\author{
Karu P.Esselle \\ School of Electrical and Data Engineering \\ University of Technology \\ Sydney, Australia \\ karu.esselle@uts.edu.au
}

\begin{abstract}
A comparative analysis of two phase correcting structures (PCSs) is presented for an electromagnetic-bandgap resonator antenna (ERA). PCSs are made out of two distinct high and low permittivity materials i.e. Rogers 03010 and polylactic acid (PLA) respectively. Design and performance analysis is based on superstrate height profile, side-lobe levels, antenna directivity, aperture efficiency, prototyping technique and cost. Insertion loss for both superstrates is greater than 0.1 $\mathrm{dB}$, assuring the maximum transmission of the antenna's radiations through the PCSs. The presented study is based on full wave analysis used to integrate sections of superstrate with custom phase-delays, to attain nearly uniform phase at the output, resulting in improved radiation performance of antenna. The peak directivity of the ERA loaded with Rogers O3010 PCS has increased by $7.3 \mathrm{~dB}$, which is $1.2 \mathrm{~dB}$ higher than that of PLA PCS. In addition, the height of the PCS made of Rogers is $71.3 \%$ smaller than the PLA PCS. However, the former will involve fabrication complexities related to machining compared to the latter which can be additively manufactured in single step.
\end{abstract}

Keywords- Aperture phase distribution, directivity enhancement, electromagnetic bandgap resonator antenna, phase correction, rapid prototyping, transmission phase.

\section{INTRODUCTION}

Electromagnetic bandgap resonator antennas (ERAs) also known as Febry-Perot cavity antennas, resonant cavity antennas or 2D leaky wave antennas have drawn unprecedented interest because of their simple configuration and non-complex feed mechanism [1], [2] . Their structure consists of resonant cavity formed between a fully reflecting ground plane and a partially reflecting structure (PRS). The PRS can be made of all-dielectric materials or 2D printed surfaces [3] - [5]. The small feed antenna mounted on the ground is typically less directive, such as slot or patch antenna. Working principle of antenna is based on condition of resonance of the cavity i.e. spacing between the ground plane and the PRS, which needs to be $\lambda_{0} / 2$, where $\lambda_{0}$ is wavelength at operating frequency [6]. Reflections inside the cavity distribute energy in lateral directions of PRS which yields improved radiation performance because of increased effective radiating aperture. It has been found in earlier investigations that phase distribution along the aperture is non-uniform, particularly for cavities with less reflective PRSs, which is a limitation in achieving the optimal antenna performance [7] - [9]. Phase correcting structure (PCS) was then proposed for EBGs to improve aperture phase distribution and enhance radiation characteristics of antenna [8]. It should be mentioned that the simultaneous manipulation of transmission magnitude and phase have recently been proposed for sidelobe improvement [10] - [12].

In this paper, the performance of two PCSs made out of PLA and Rogers O3010 materials referred to as PCSPLA and PCS3010, respectively, are compared. For a particular range of dielectric heights, transmission characteristics of both materials were explored to get information related to amplitude and propagation phase. For both designs, unit-cell dielectric height values with insertion loss $>0.1 \mathrm{~dB}$ were considered to make PCSs highly transmitting. Full-wave numerical electromagnetic solver CST Microwave Studio was used for a simulation-based analysis. The non-uniformity of the antenna phase distribution is quantified on an imaginary plane parallel to the antenna aperture. The phase error is then recorded in different sections to determine the total phase range required for the phase compensation. Next, appropriate

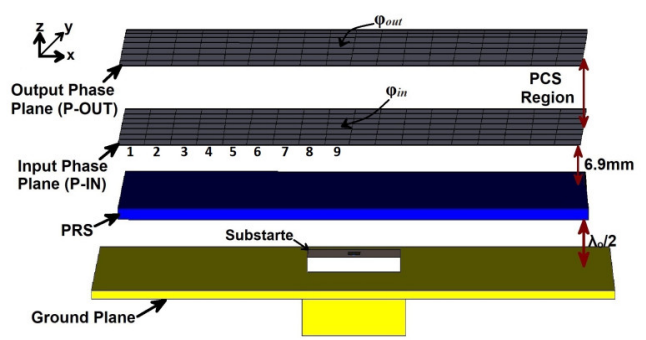

Fig. 1. Layout of PCS with ERA 
dielectric heights were integrated in different sections to form two PCSs. Computed results of these phase-corrected and classical 1D ERA are presented, and the key parameters, such as peak directivity, bandwidth and aperture efficiency are compared.

The rest of the paper is organized such that section II explains general configuration of RCA and PCS. Section III is related to study of transmission characteristics of materials used for PCSs design. Section IV is about the performance comparison with PCSPLA and PCS3010. Conclusion is documented in Section V.

\section{PCS WITH ERA LAYOUT}

Fig. 1 shows the configuration of the ERA designed to operate at $20 \mathrm{GHz}$. A dielectric slab with a permittivity of 4.5 and loss tangent of 0.002 (Rogers TMM4) acts as PRS and is placed at a height of $7.7\left(\approx \lambda_{\mathrm{o}} / 2\right)$ from the ground plane. The PRS slab has square geometry with maximum lateral size of $90 \mathrm{~mm}$ and height of $1.24 \mathrm{~mm}$, respectively, and the area of square shaped ground plane is $(120 \mathrm{~mm})^{2}$. To retrieve information related to non-uniform phase distribution on the aperture, a hypothetical plane is considered $6.9 \mathrm{~mm}$ above the PRS, illustrated as Input Phase Plane (P-IN) in Fig. 1. Another hypothetical plane is considered parallel to P-IN referred to as Phase output plane (P-OUT), where a relatively planar phase response is expected after introduction of the PCS between PIN and P-OUT. A grid of $18 \times 18$ cells is considered on P-IN to record dominant component of E-field at discrete points above the aperture. Each unit cell in grid has square geometry with a maximum dimension of $5.0 \mathrm{~mm}$. The input phase values $\left(\varphi_{\text {in }}\right)$ are recorded at the center point of each grid cell to infer nonuniformity of phase in P-IN, as explained in [13]. To attain a uniform phase distribution on the P-OUT, an arbitrary constant value greater than the total phase error on aperture is defined for the output phase $\left(\varphi o u t=283^{\circ}\right)$. Thus, the required phase shift for each unit cell can be calculated by

$$
\varphi_{\text {req }}=\varphi_{\text {out }}-\varphi_{\text {in }}
$$

Therefore, for each column a specific amount of phase shift $\left(\varphi_{\text {req }}\right)$ should be provided by the PCS to attain planar response at P-OUT. Due to the circular symmetry of phase distribution on xy-plane of ERAs aperture, only 9 distinct phase transformers are needed to compensate phase differences on the aperture [14], [15]. Phase shift offered by materials depends on their transmission characteristics, which have been discussed in detail for both materials of interest (PLA and RogersO3010) in the next section.

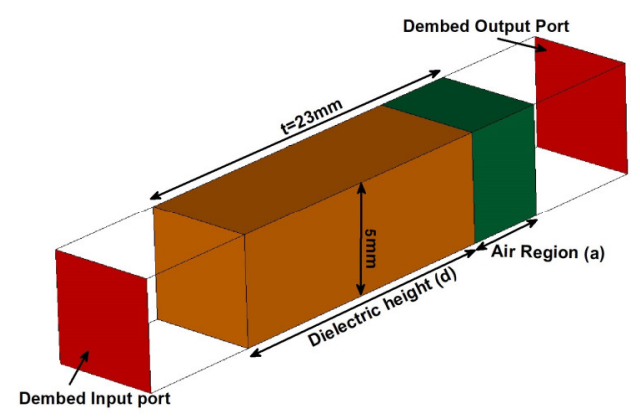

Fig. 2. Unit-cell configuration for PCS design.

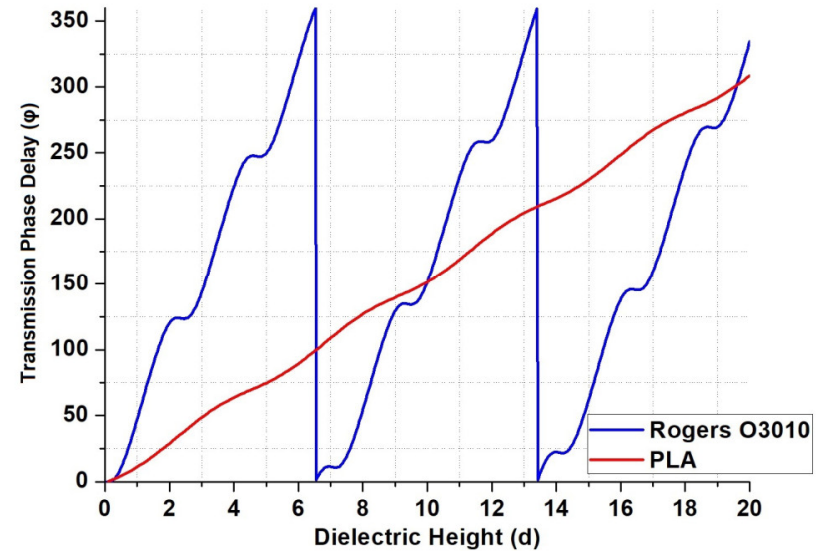

Fig. 3. Wrapped phase plots for PLA and Rogers O3010 materials against variation of height.

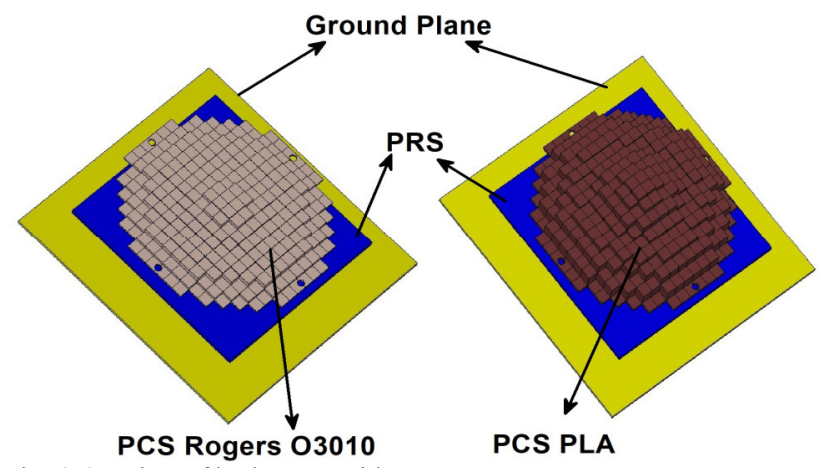

Fig. 4. 3D view of both PCSs with ERA.

\section{MATERIAl ChARACTERISTICS AND PCS DESIGN}

Fig. 2. Shows a unit cell of the grids between P-IN and POUT to provide desired phase delay. It has periodicity of 5.0 $\mathrm{mm}$, total height of $23 \mathrm{~mm}$ and partially comprises of dielectric and air regions. Thus, dielectric and air region will combine to provide required phase delay in the PCS configuration. Transmission amplitude and phase delay through air and dielectric region can be expressed as [14]:

$$
\begin{gathered}
T=T_{\text {air }}+T_{\text {dielectric }} \\
T=k_{o}(d-t)+\frac{\tau_{1} \tau_{2} e^{-j k_{1} d}}{1+\rho_{1} \rho_{2} e^{-2 j k_{1} d}}
\end{gathered}
$$

Above equation yields a complex number whose real and imaginary parts represent amplitude and phase of transmission coefficient through each unit cell. While, designing PCSs the unit-cells with amplitude values $>0.1 \mathrm{~dB}$ were considered to make the structures highly transmitting. Using eq. (3) parameter h was swept from $0.1 \mathrm{~mm}$ to $20 \mathrm{~mm}$ for dielectric constant of 2.72 (PLA) and 10.2 (Rogers O3010) to extract transmission phase-delay for various heights, as shown in Fig. 3 . Table I lists $9 \varphi_{\text {req }}$ values required for equivalent grid cells

Table I. phase values and corresponding heights for both PCSs

\begin{tabular}{|c|c|c|c|c|}
\hline $\begin{array}{c}\text { grid } \\
\text { cell no. }\end{array}$ & $\varphi_{\text {in }}$ & $\varphi_{\text {req }}$ & $\begin{array}{c}\text { Height PLA } \\
\text { from Fig. 3 }\end{array}$ & $\begin{array}{c}\text { Height RO3010 } \\
\text { Fig. 3 }\end{array}$ \\
\hline 1 & 273.12 & 9.88 & 18.3 & 5.56 \\
\hline 2 & 204.28 & 78.72 & 16.7 & 5.25 \\
\hline 3 & 144.26 & 138.74 & 14.8 & 4.02 \\
\hline 4 & 116.48 & 166.52 & 11.9 & 3.53 \\
\hline 5 & 124.27 & 158.73 & 10.4 & 3.2 \\
\hline 6 & 96.66 & 186.34 & 10.9 & 3.3 \\
\hline 7 & 57.39 & 225.61 & 8.9 & 2.92 \\
\hline 8 & 21.4 & 261.6 & 5.3 & 1.37 \\
\hline 9 & 0 & 283 & 0.9 & 0.49 \\
\hline
\end{tabular}




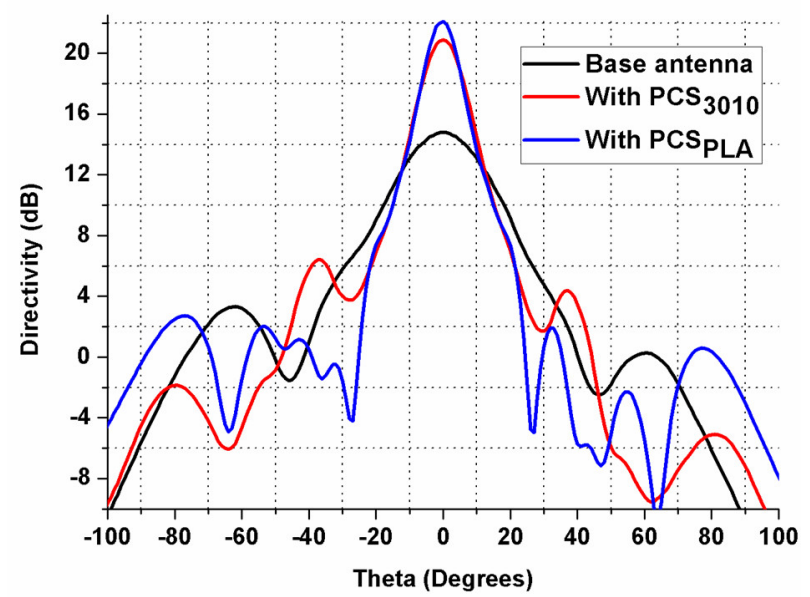

Fig. 5: Directivity plot for base ERA and PCS loaded ERAs at 20 $\mathrm{GHz}$.

to attain $\varphi_{\text {out }}=283^{\circ}$ at each section in P-OUT. Corresponding phase values were selected from the plot data to make two different PCSs, as shown in Fig. 4. Table I also lists corresponding heights of unit-cells to attain the desired phase shift at various sections of both PCSs.

\section{COMPARISON OF PCSS}

Radiation patterns of the antenna with and without the PCSs at operating frequency $(20 \mathrm{GHz})$ are shown in Fig. 5. It is evident that the peak directivity of $22.1 \mathrm{~dB}$ is achieved for the ERA with PCS3010, which is $7.3 \mathrm{~dB}$ more than that of the bare ERA. The PCSPLA mounted ERA yields peak directivity of $20.9 \mathrm{~dB}$, thereby resulting in $6.1 \mathrm{~dB}$ improvement in the peak directivity of the base ERA. So, PCS3010 offers $1.2 \mathrm{~dB}$ higher directivity than that of PCSPLA. Directivity plots for frequency range $19 \mathrm{GHz}$ to $21.5 \mathrm{GHz}$ are shown in Fig. 6. The most extensive $3-\mathrm{dB}$ directivity bandwidth is demonstrated by the base ERA alone, which is, $10.8 \%$ while the smallest bandwidth is for the ERA with PCS3010 7.33\%. Whereas, in case of ERA with PCSPLA, reasonably large bandwidth of $10.7 \%$ is achieved, making the cavity suitable for wideband operation even though the PCSs were designed to operate at $20 \mathrm{GHz}$. From Fig. 5, in terms of quality of the radiation patterns, the side lobe levels for the base ERA are the worst ($11.5 \mathrm{~dB})$ and the best for the base ERA with PCSPLA (-19.4

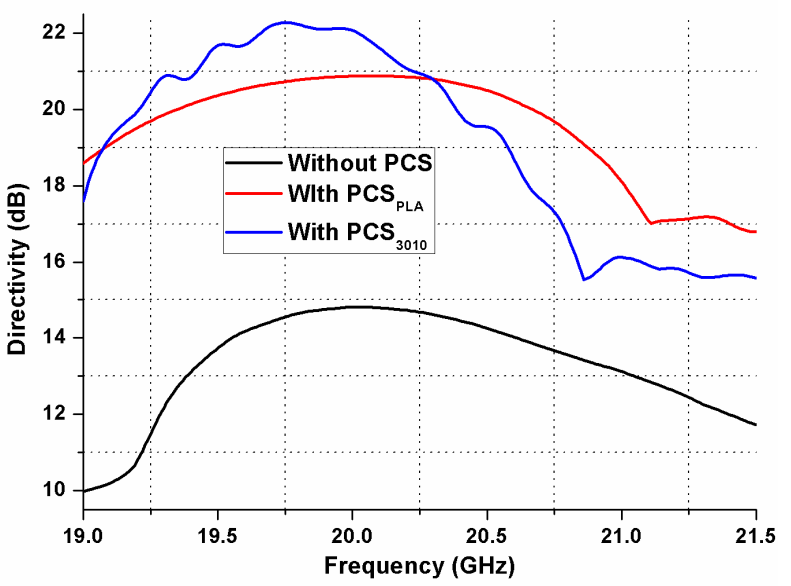

Fig. 6: Wideband directivity plots for base ERA and PCS loaded ERAs
Table I. Comparison between base ERA, PCS3010 loaded ERA and

\begin{tabular}{|c|c|c|c|}
\multicolumn{5}{c}{ PCSPLA loaded ERA } \\
\hline Type & Base ERA & With PCS S010 $_{2}$ & With PCS $_{\text {PLA }}$ \\
\hline Directivity (dB) & 14.8 & 22.1 & 20.9 \\
\hline $\begin{array}{c}\text { 3-dB dir. BW } \\
(\%)\end{array}$ & 10.8 & 7.3 & 10.7 \\
\hline SLL (dB) & -11.5 & -14.8 & -19.4 \\
\hline AE (\%) & 46.6 & 35.06 & 9.01 \\
\hline Cost & - & High & Low \\
\hline Fabrication & - & $\begin{array}{c}\text { Subtractive } \\
\text { manufacturing }\end{array}$ & $\begin{array}{c}\text { Additive } \\
\text { manufacturing }\end{array}$ \\
\hline Height (mm) & - & 21.8 & 34.85 \\
\hline
\end{tabular}

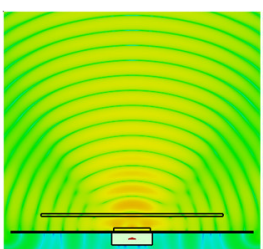

(a)

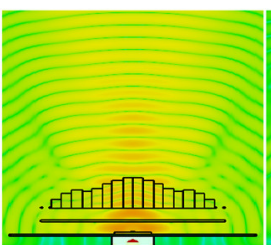

(b)

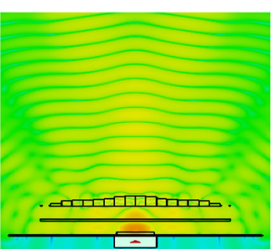

(c)
Fig. 7: Dominant E-field component above aperture for all three ERAs (a) Base (b) PCSPLA loaded and (c) PCS3010 loaded.

dB). Both phase-corrected ERAs have comparably good side lobe levels compared to the bare ERA, as ERA with PCS3010 has side lobe levels of $-14.8 \mathrm{~dB}$.

The aperture efficiency of the ERA with PCS3010 is $46.6 \%$ for the simulated gain of $21.73 \mathrm{~dB}$, while it is $35.06 \%$ for the ERA with PCSPLA. Aperture efficiency with PCSs is high because the effective radiating aperture is more uniform, due to phase correction depicted in Fig. 7. The smallest aperture efficiency is $9.01 \%$ for the base ERA due to its nonuniform radiating aperture. Finally, the key performance parameters of the ERAs are summarized in Table 1. Overall, the phase-corrected ERA with PCS3010 has the best performance in terms of peak directivity, directivity bandwidth and aperture efficiency. The ERA loaded with PCS3010 has a total height of $21.8 \mathrm{~mm}(1.45 \lambda \mathrm{o}$ at $20 \mathrm{GHz})$ which is considerably smaller than the ERA with PCSPLA, i.e. $34.85 \mathrm{~mm}(2.3 \lambda \mathrm{o}$ at $20 \mathrm{GHz})$.

In terms of fabrication and cost, the PCSPLA can be 3D printed using most commonly available, low cost PLA filament. Hence it can be quickly prototyped using additive manufacturing. Whereas, PCS3010 involves traditional subtractive manufacturing, which is cumbersome as it requires a lengthy $\mathrm{CNC}$ machining. Moreover, 3D printable polymers show performance comparison with RF laminates despite being low cost. Hence PCSPLA is more costeffective.

\section{CONCLUSION}

A detailed performance comparison of two phase corrected and base ERA has been presented. According to the numerically calculated radiation patterns, significant improvement is achieved in directivity, aperture efficiency and side lobe levels, as a result of phase correction carried out by the PCSs. It has been shown that the height of PCS made of high permittivity material is $71.3 \%$ shorter than that of the low permittivity PCS. Low permittivity PCS is cost effective and can be rapidly prototyped through additive manufacturing contrary to high permittivity PCS, which will require subtractive manufacturing. 


\section{REFERENCES}

[1] C. Cheype, C. Serier, M. Thevenot, T. Moneiere, A. Reineix and B. Jecko, "An electromagnetic bandgap resonator antenna," IEEE Trans. Antennas Propag., vol. 50, pp. 1285-1290, 2002.

[2] Y. Ge, Z. Sun, Z. Chen and Y.-Y. Chen, "A High-Gain Wideband LowProfile Fabry-Perot Resonator Antenna With a Conical Short Horn," IEEE Antennas Wireless Propag. Lett., vol. 15, pp. 1889-1892, 2016.

[3] A. Lalbakhsh, M. U. Afzal, K. P. Esselle, S. L. Smith and B. A. Zeb, "Single-dielectric Wideband Partially Reflecting Surface with Variable Reflection Components for Realization of a Compact High-gain Resonant Cavity Antenna," IEEE Transactions on Antennas and Propagation, vol. 76, pp. 1916-1921, 2019.

[4] K. Konstantinidis, A. P. Feresidis and P. S. Hall, "Multilayer partially reflective surfaces for broadband Fabry-Perot cavity antennas," IEEE Transactions on Antennas and Propagation, vol. 62, pp. 3474-3481, 2014.

[5] A. Lalbakhsh, M. U. Afzal, K. P. Esselle and S. L. Smith, "A High-gain Wideband EBG Resonator Antenna for $60 \mathrm{GHz}$ Unlicenced Frequency Band," Accepted in Eucap, London, 2018.

[6] G. V. Trentini, "Partially reflecting sheet arrays," IEEE Trans. Antennas Propagat., vol. 4, pp. 666-671, 1956.

[7] L. Zhou, X. Chen, X. Duan and J. Li, "Fabry-Perot antenna using a threelayer phase shifting structure for gain enhancement," IET Microwaves, Antennas $\backslash \&$ Propagation, vol. 12, pp. 400-405, 2017.

[8] M. U. Afzal, K. P. Esselle and B. A. Zeb, "Dielectric phase-correcting structures for electromagnetic band gap resonator antennas," IEEE Trans. Antennas Propag., vol. 63, pp. 3390-3399, 2015.
[9] T. Hayat, K. P. Esselle, M. U. Afzal and K. Singh, "3D Printed All Dielectric Phase Correcting Surface for Resonant Cavity Antenna," in 2018 IEEE Asia-Pacific Conference on Antennas and Propagation (APCAP), 2018.

[10] K. Dutta, D. Guha and C. Kumar, "Synthesizing aperture fields over the superstrate of resonance cavity antenna for modifying its radiation properties," IEEE Antennas and Wireless Propagation Letters, vol. 15, pp. 1677-1680, 2016.

[11] A. Lalbakhsh, M. U. Afzal, K. P. Esselle and S. L. Smith, "Wideband Near-Field Correction of a Fabry-Perot Resonator Antenna," IEEE Transactions on Antennas and Propagation, vol. 67, pp. 1975-1980, 2019.

[12] K. Dutta, D. Guha and C. Kumar, "Theory of Controlled Aperture Field for Advanced Superstrate Design of a Resonance Cavity Antenna With Improved Radiations Properties," IEEE Transactions on Antennas and Propagation, vol. 65, pp. 1399-1403, 2017.

[13] A. Lalbakhsh, M. U. Afzal and K. P. Esselle, "Multi-objective Particle Swarm Optimization to Design a Time Delay Equalizer Metasurface for an Electromagnetic Band Gap Resonator Antenna," IEEE Antennas Wireless Propag. Lett., vol. 16, pp. 912-915, 2017.

[14] T. Hayat, M. U. Afzal, A. Lalbakhsh and K. P. Esselle, "3D Printed Phase-Rectifying Transparent Superstrate for Resonant-Cavity Antenna," IEEE Antennas and Wireless Propagation Letters, 2019.

[15] T. Hayat, M. U. Afzal, A. Lalbakhsh and K. P. Esselle, "Additively Manufactured Perforated Superstrate to Improve Directive Radiation Characteristics of Electromagnetic Source," IEEE Access, 2019. 